\title{
La Gestión de procesos internos en las empresas prestadoras de servicio de agua y alcantarillado en el Perú
}

\author{
Mg. Cieza Pérez, Alberto \\ cipereza@ucvvirtual.edu.pe \\ https://orcid.org/0000-0002-4144-0948 \\ Msc. Mirtha Culqui Lozada \\ clozadam@ucvvirtual.edu.pe \\ https://orcid.org/0000-0002-8780-9755
}

Mg. Víctor Hugo Puican Rodríguez

Victor-puican@hotmail.com https://orcid.org/0000-0001-7402-9576

Dr. Marcelino Callao Alarcón calarconm@ucvvirtual.edu.pe $\underline{\text { https://orcid.org/000-0001-7295-2375 }}$

Universidad César Vallejo

Chiclayo - Perú

\section{RESUMEN}

Se llevó a cabo una revisión documental sobre la producción y publicación de trabajos de investigación referentes al estudio de la variable Gestión de procesos internos en las empresas prestadoras de servicio de agua y alcantarillado en Latinoamérica. El propósito del análisis bibliométrico propuesto en el presente documento, es conocer las características principales del volumen de publicaciones registradas en base de datos Scopus durante el periodo 2015-2020 en países Latinoamericanos, logrando la identificación de 27 publicaciones en total. La información suministrada por dicha plataforma, fue organizada mediante gráficos y figuras categorizando la información por el Año de Publicación, País de Origen, Are de Conocimiento y Tipo de Publicación. Una vez descritas dichas características, se referencia mediante un análisis cualitativo, la postura de diferentes autores frente a la temática propuesta. Dentro de los principales 
hallazgos realizados por medio de la presente investigación, se encuentra que Colombia con 16 publicaciones, es el país latinoamericano con mayor producción. El Área de Conocimiento que mayor aporte hizo a la construcción de material bibliográfico referente al estudio de La Gestión de procesos internos en las empresas prestadoras de servicio de agua y alcantarillado fue ingeniería con 14 documentos publicados, y el Tipo de Publicación que más fue usado durante el periodo señalado anteriormente fue el Articulo de Revista que representan el 74\% de la producción científica total.

Palabras clave: gestión procedimental; empresas de agua y alcantarillado 


\title{
Management of internal processes in water and sewage utilities in Peru
}

\begin{abstract}
A documentary review was carried out on the production and publication of research papers related to the study of the variable Internal Process Management in water and sewerage service providers in Latin America. The purpose of the bibliometric analysis proposed in this document, is to know the main characteristics of the volume of publications registered in Scopus database during the period 2015-2020 in Latin American countries, achieving the identification of 27 publications in total. The information provided by said platform, was organized by means of graphs and figures categorizing the information by Year of Publication, Country of Origin, Area of Knowledge and Type of Publication. Once these characteristics were described, the position of different authors regarding the proposed topic was referenced by means of a qualitative analysis. Among the main findings of this research, it is found that Colombia, with 16 publications, is the Latin American country with the highest production. The area of knowledge that made the greatest contribution to the construction of bibliographic material related to the study of internal process management in water and sewerage service providers was engineering with 14 published documents, and the type of publication that was most used during the period mentioned above was the journal article, which represents $74 \%$ of the total scientific production.
\end{abstract}

Key words: procedural management; water and sewerage companies.

Artículo recibido: 05 octubre. 2021 Aceptado para publicación: 02 noviembre 2021 Correspondencia: cipereza@ucvvirtual.edu.pe Conflictos de Interés: Ninguna que declarar 


\section{INTRODUCCIÓN}

Los procesos internos de las empresas de acueducto y de alcantarillado son de gran importancia para garantizar la calidad del servicio de agua potable y de tratamiento de aguas residuales, ya que de estos procesos depende la gestión técnica de las empresas. Dependiendo de los procesos internos que se acojan en estas empresas se definen las políticas que se implementan para el correcto tratamiento en las empresas de acueducto y alcantarillado. Un ejemplo de cambio de los procesos internos se evidencia en el estudio llamado "Las empresas de agua dulce. Proyectos privados para sistemas de abastecimiento de agua potable en el Perú y México" (Birrichaga Gardida, 2006) el cual hace un recorrido en los cambios que se han hecho en los procedimientos que se emplean en las empresas de acueducto como manera de aumentar la eficiencia de los procesos técnicos para garantizar la calidad del servicio que prestan a la comunidad, en este caso se garantiza el correcto tratamiento del agua potable.

Teniendo conocimiento de la importancia de esta gestión interna en las empresas de acueducto y alcantarillado, en Perú se buscan emplean nuevas medidas que garanticen la calidad en la prestación de este servicio. Por ejemplo, "Problemática en la composición del Directorio en una Empresa Prestadora de Servicios de Saneamiento ubicada en el centro oeste del Perú" (García Rivera, 2020) en el que se busca determinar los problemas de estas empresas en los procedimientos administrativos con el fin de innovar los procesos internos de las empresas de saneamiento teniendo un cambio en la composición del Directorio y en el procedimiento de elección de sus miembros administrativos con el fin de garantizar una buena gestión y que opta por la sostenibilidad empresarial como manera de ofrecer este servicio de manera permanente en las localidades urbanas del oeste de Perú. Por lo anterior, es importante conocer en materia de recursos bibliográficos, el estado actual de la investigación referente a La Gestión de procesos internos en las empresas prestadoras de servicio de agua y alcantarillado, por lo que se propone un análisis bibliométrico de la producción científica registrada en base de datos Scopus durante el periodo 2015-2020 que permita dar respuesta a la pregunta ¿ Cómo ha sido la producción y publicación de trabajos de investigación relacionados al estudio de la variable Gestión de procesos internos en las empresas prestadoras de servicio de agua y alcantarillado durante el periodo 2015-2020? 


\section{ESTRATEGIAS METODOLÓGICAS O MATERIALES Y MÉTODOS}

\subsection{Objetivo general}

- Analizar desde una perspectiva bibliométrica y bibliográfica, la producción de trabajos de investigación de alto impacto sobre la variable La Gestión de procesos internos en las empresas prestadoras de servicio de agua y alcantarillado durante el periodo 2015-2020.

\subsection{Metodología}

Se realiza análisis cuantitativo de la información suministrada por Scopus bajo un enfoque bibliométrico sobre la producción científica referente a La Gestión de procesos internos en las empresas prestadoras de servicio de agua y alcantarillado. Asimismo, se analiza desde una perspectiva cualitativa, ejemplos sobre algunos trabajos de investigación publicados en el área de estudio señalada anteriormente, desde un enfoque bibliográfico para describir la postura de diferentes autores frente al tema propuesto.

La búsqueda se realiza a través de la herramienta suministrada por Scopus y se establecen parámetros referenciados en la Tabla 1.

\subsection{Diseño metodológico}

Tabla1. Diseño metodológico.

\begin{tabular}{|c|c|c|c|}
\hline & FASE & DESCRIPCION & CLASIFICACION \\
\hline FASE 1 & $\begin{array}{l}\text { Levantamiento de } \\
\text { datos }\end{array}$ & $\begin{array}{l}\text { Se realiza el } \\
\text { levantamiento de } \\
\text { datos por medio de la } \\
\text { herramienta Búsqueda } \\
\text { en la página web de } \\
\text { Scopus, por medio de } \\
\text { la cual se logra la } \\
\text { identificación de un } \\
\text { total de } \\
\text { publicaciones. }\end{array}$ & $\begin{array}{l}\text { Documentos } \\
\text { publicados cuyas } \\
\text { variables de estudio } \\
\text { estén relacionadas a La } \\
\text { Gestión de procesos } \\
\text { internos en las } \\
\text { empresas prestadoras } \\
\text { de servicio de agua y } \\
\text { alcantarillado. } \\
\text { Trabajos } \\
\text { investigación } \\
\text { publicados durante el } \\
\text { Periodo 2015-2020. } \\
\text { Limitado a países } \\
\text { latinoamericanos. } \\
\text { Sin distinción de área } \\
\text { de conocimiento. } \\
\text { Sin distinción de tipo } \\
\text { de publicación. }\end{array}$ \\
\hline
\end{tabular}




\begin{tabular}{|c|c|c|c|}
\hline FASE 2 & $\begin{array}{l}\text { Construcción de } \\
\text { material de análisis }\end{array}$ & $\begin{array}{l}\text { Se procede a la } \\
\text { organización de la } \\
\text { información } \\
\text { identificada en la fase } \\
\text { anterior. } \\
\text { clasificación se hará } \\
\text { mediante gráficos, } \\
\text { figuras y tablas a } \\
\text { partir de datos } \\
\text { suministrados por } \\
\text { Scopus. }\end{array}$ & $\begin{array}{l}\text { Co-ocurrencia de } \\
\text { Palabras. } \\
\text { Año de publicación } \\
\text { País de origen de la } \\
\text { publicación. } \\
\text { Área de conocimiento. } \\
\text { Tipo de Publicación }\end{array}$ \\
\hline FASE 3 & $\begin{array}{lr}\text { Redacción de las } \\
\text { conclusiones } \\
\text { documento final }\end{array}$ & $\begin{array}{l}\text { Luego del análisis } \\
\text { realizado en la fase } \\
\text { anterior, se procede a } \\
\text { la redacción de las } \\
\text { conclusiones } \\
\text { elaboración } \\
\text { documento final. }\end{array}$ & \\
\hline
\end{tabular}

Fuente: Elaboración propia (2021)

\section{RESULTADOS}

\subsection{Co-ocurrencia de palabras}

La Figura 1 muestra la co-ocurrencia de palabras clave dentro de las publicaciones identificadas en base de datos Scopus.

\section{Figura 1. Co-ocurrencia de palabras}

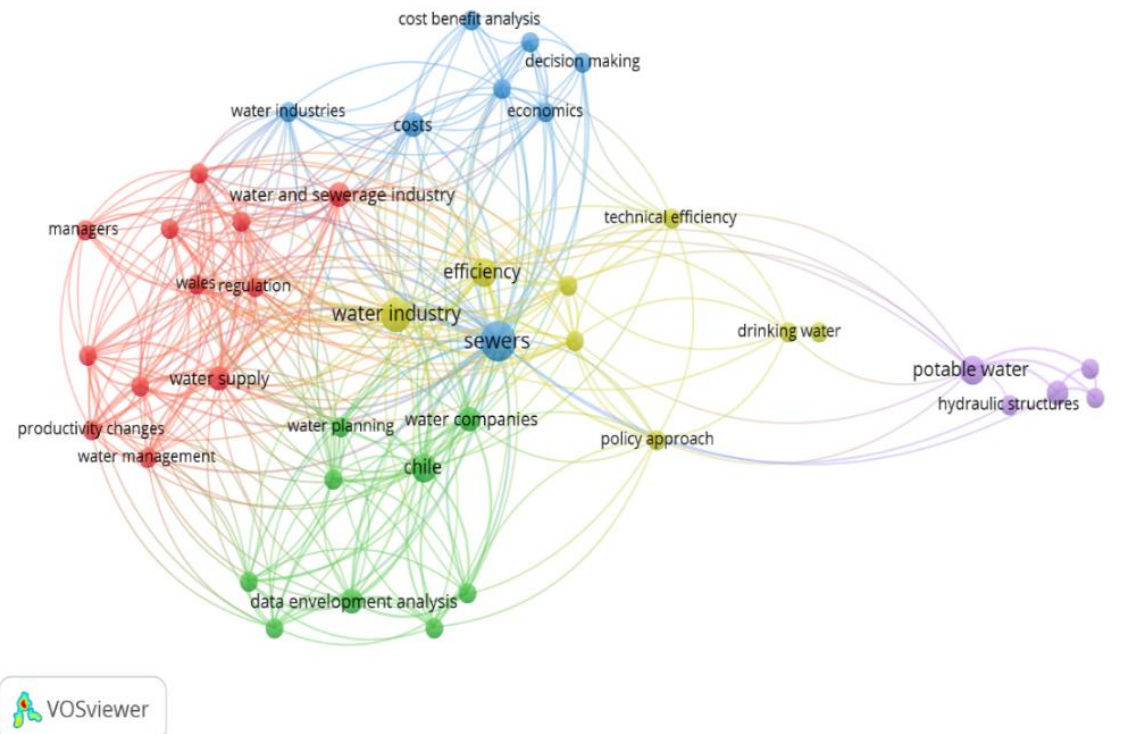

Fuente: Elaboración propia (2021); a partir de datos suministrados por Scopus. 
Como lo muestra la figura 1, alcantarillado es la palabra clave más utilizadas en las investigaciones que tienen relación con las variables en estudio, ya que esta palabra clave está relacionada con la naturaleza de las empresas que se estudian en esta investigación. También encontramos palabras claves afines como industria del agua y empresas de acueducto las cuales hacen referencia al estudio de La Gestión de procesos internos en las empresas prestadoras de servicio de agua y alcantarillado. En materia procedimental, encontramos palabras claves como análisis de costos y beneficios, gestión de acueducto, gestión de políticas y administración las cuales nos indican los procedimientos que se generan en las empresas de acueducto de manera interna para la optimización de la prestación de los servicios y para asegurar la eficiencia técnica de las plataformas usadas para el tratamiento de las aguas para el suministro de agua potable de los complejos urbanos.

\subsection{Distribución de la producción científica por año de publicación.}

La Figura 2 muestra como está distribuida la producción científica según el año de publicación, teniendo en cuenta que se toma el periodo comprendido entre los años 2015 y 2020.

Figura 2. Distribución de la producción científica por año de publicación.

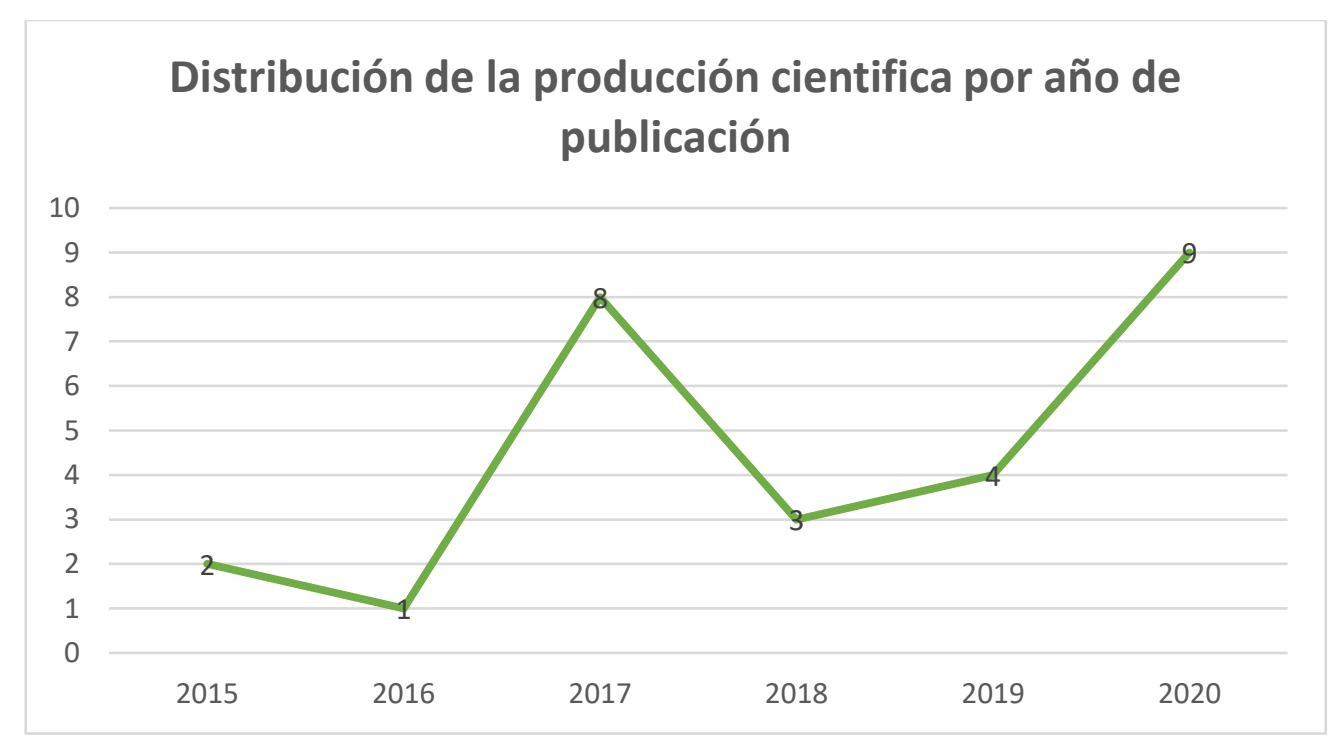

Fuente: Elaboración propia (2021); a partir de datos suministrados por Scopus.

Como se muestra en la figura 2, el 2020 es el año con mayor número de publicaciones registradas teniendo un total de 9 publicaciones, dentro de las cuales se encuentra "Propuesta de metamodelo para la regulación de la demanda urbana de agua potable" (Lizcano-Caro, Medina-Daza, \& González-Pérez, 2020) en este estudio se propuso un 
metamodelo de demanda urbana de agua potable en escenarios de equilibrio inestable, con criterios solidarios y priorización de inversiones eco ambientales a través de la optimización de los procesos internos de las empresas prestadoras de este servicio, además de implementar el sistema de apoyo a la toma de decisiones con la ayuda de un análisis de redes neuronales se adapta mejor a las condiciones de los complejos urbanos ayudando a conocer las necesidades dependiendo de las situación socioeconómica de la comunidad que se estudia.

El 2019 registra 4 publicaciones y el año 2018 tiene un total de 3 documentos. El 2017 con 8 documentos es el segundo año con mayor número de publicaciones registradas en Scopus, dentro de estos se encuentra el artículo titulado "Descomposición del crecimiento de la productividad de las empresas de agua y alcantarillado: un enfoque empírico para Chile" (Molinos-Senante \& Sala-Garrido, 2017) esta investigación propone La evaluación del crecimiento de la productividad de las empresas de agua y alcantarillado como la herramienta para garantizar su sostenibilidad a largo plazo y proteger los intereses de los clientes. Por lo que desde una perspectiva política y gerencial, este estudio revela la importancia de cuantificar la contribución de los insumos y productos en el crecimiento de la productividad para apoyar la toma de decisiones y la innovación de los procesos internos que se realizan en estas empresas para la optimización de la prestación de sus servicios.

\subsection{Distribución de la producción científica por país de origen.}

La Figura 3 muestra cómo está distribuida la producción científica según la nacionalidad de los autores.

Figura 3. Distribución de la producción científica por país de origen.

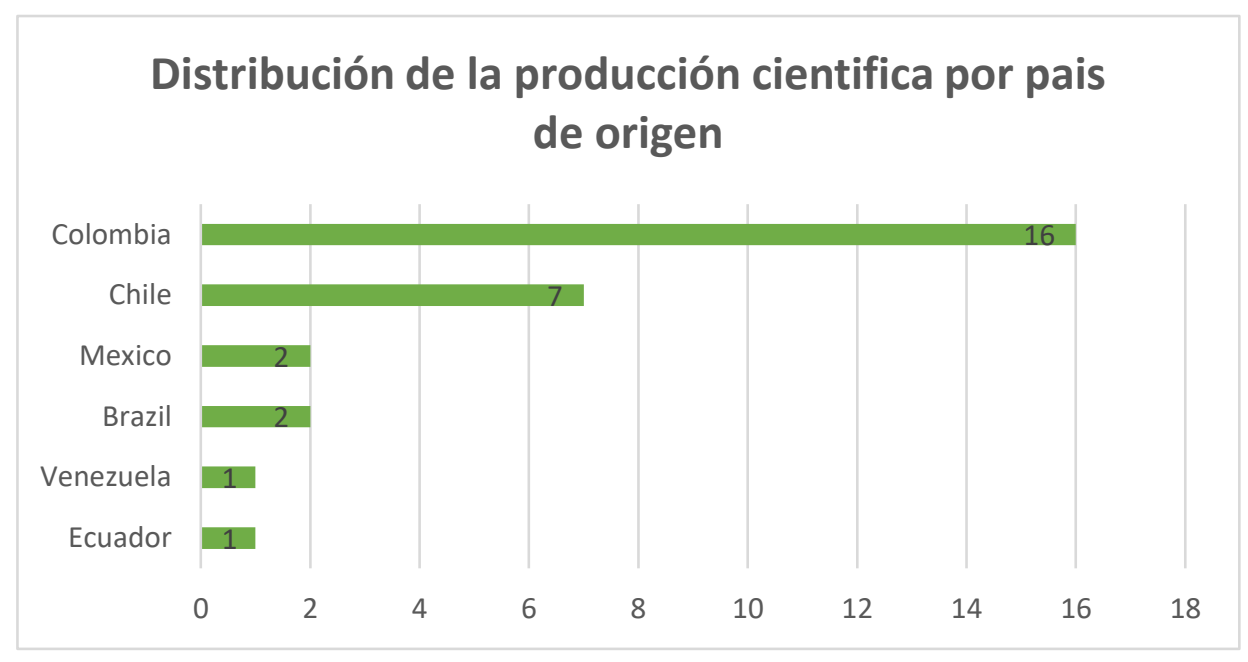

Fuente: Elaboración propia (2021); a partir de datos suministrados por Scopus. 
Colombia es el país con mayor aporte a investigaciones relacionadas a La Gestión de procesos internos en las empresas prestadoras de servicio de agua y alcantarillado con un total de 16 publicaciones dentro de las cuales se identifica "Optimización combinada del mantenimiento y el enrutamiento para la limpieza de aguas residuales a gran escala" (Fontecha, y otros, 2019) este estudio se enfoca en abordar el problema de planificar y programar operaciones de mantenimiento basadas en un patrón de deterioro para un conjunto de sitios distribuidos geográficamente, sujetos a fallas imprevistas, toda esta organización se hará ante procesos administrativas. En este estudio se emplea un modelo de mantenimiento impulsado por la distribución de probabilidad del tiempo entre fallas determina el momento óptimo para realizar las operaciones de mantenimiento para cada sitio dando como resultado la reducción el costo por unidad de tiempo en aproximadamente un $18 \%$ de diferencia con la empresa que presta el servicio en Bogotá. En este punto cabe resaltar que la producción de publicaciones científicas al ser clasificada por país de origen, presenta una característica especial y es la colaboración entre autores con diferente afiliación a instituciones tanto públicas como privadas, y estas instituciones pueden ser del mismo país o de diferentes nacionalidades por lo que la producción de un artículo con coautoría de diferentes autores de distintos países de origen permite a cada uno de los países sumar como una unidad en la publicaciones generales. Lo anterior se explica de una mejor manera en la Figura 4 donde se observa el flujo de trabajos en colaboración de diferentes países.

Figura 4. Co-citaciones entre países.

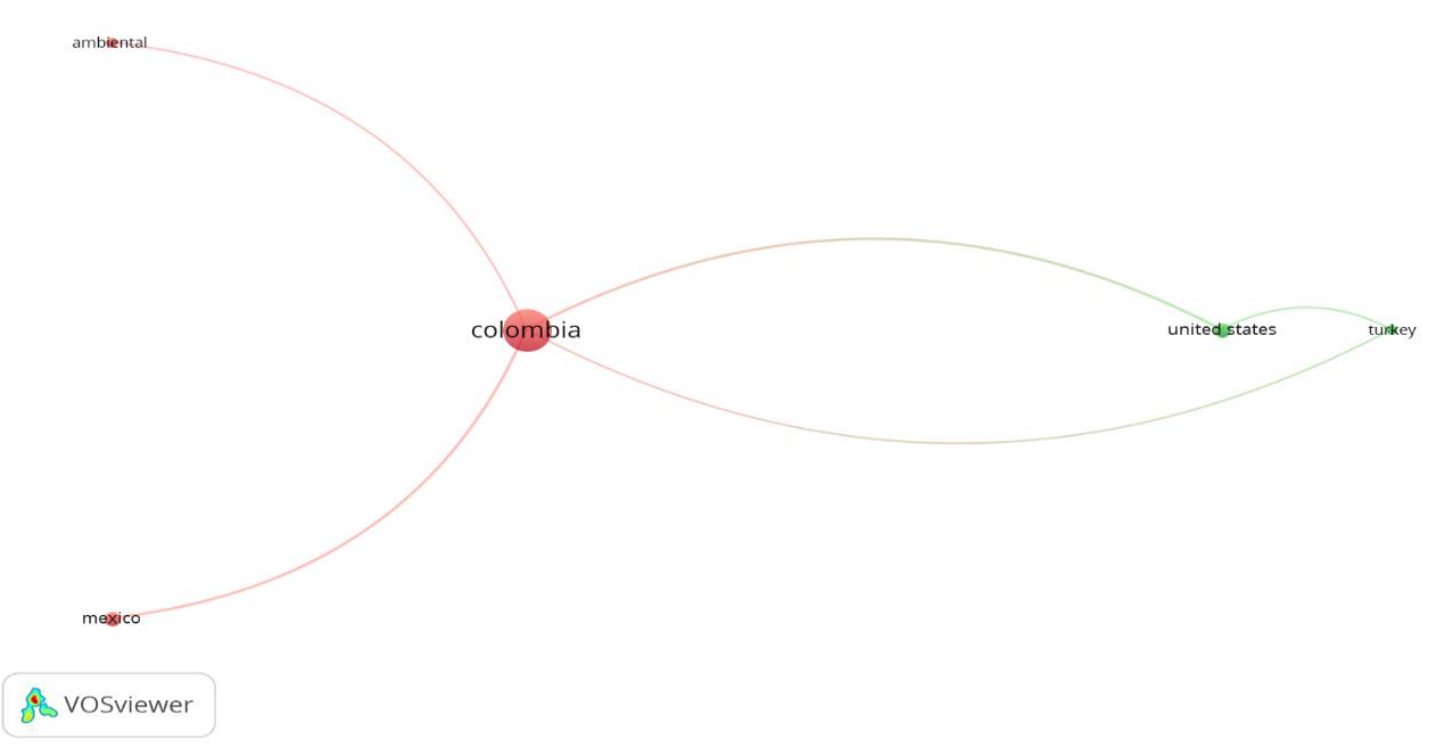

Fuente: Elaboración propia (2021); a partir de datos suministrados por Scopus 
Como se mencionó anteriormente el país con más publicaciones registradas relacionadas con la variable en estudio es Colombia, el cual también tiene colaboración con autores afiliado a instituciones de países que no pertenecen a Latinoamérica como Estado Unidos y Turquía. Chile es el segundo país con mayor participación en la investigación referente a La Gestión de procesos internos en las empresas prestadoras de servicio de agua y alcantarillado con un total de 7 documentos registrados en Scopus, dentro de estas publicaciones podemos identificar "Características tecnológicas y operativas de la industria chilena de agua y alcantarillado: una comparación de empresas públicas, concesionarias y privadas" (Molinos-Senante \& Maziotis, 2020) este estudio considera la evaluación de las características tecnológicas y operativas de la industria del agua y el alcantarillado es interesante para los investigadores y los formuladores de políticas que optimicen los procesos internos de las empresas prestadoras de ese servicio .

\subsection{Distribución de la producción científica por área de conocimiento}

A continuación, se muestra en la Figura 5, como está distribuida la producción de publicaciones científicas según el área de conocimiento por medio de la cual se ejecutan las diferentes metodologías de investigación.

Figura 5. Distribución de la producción científica porarea de conocimiento.

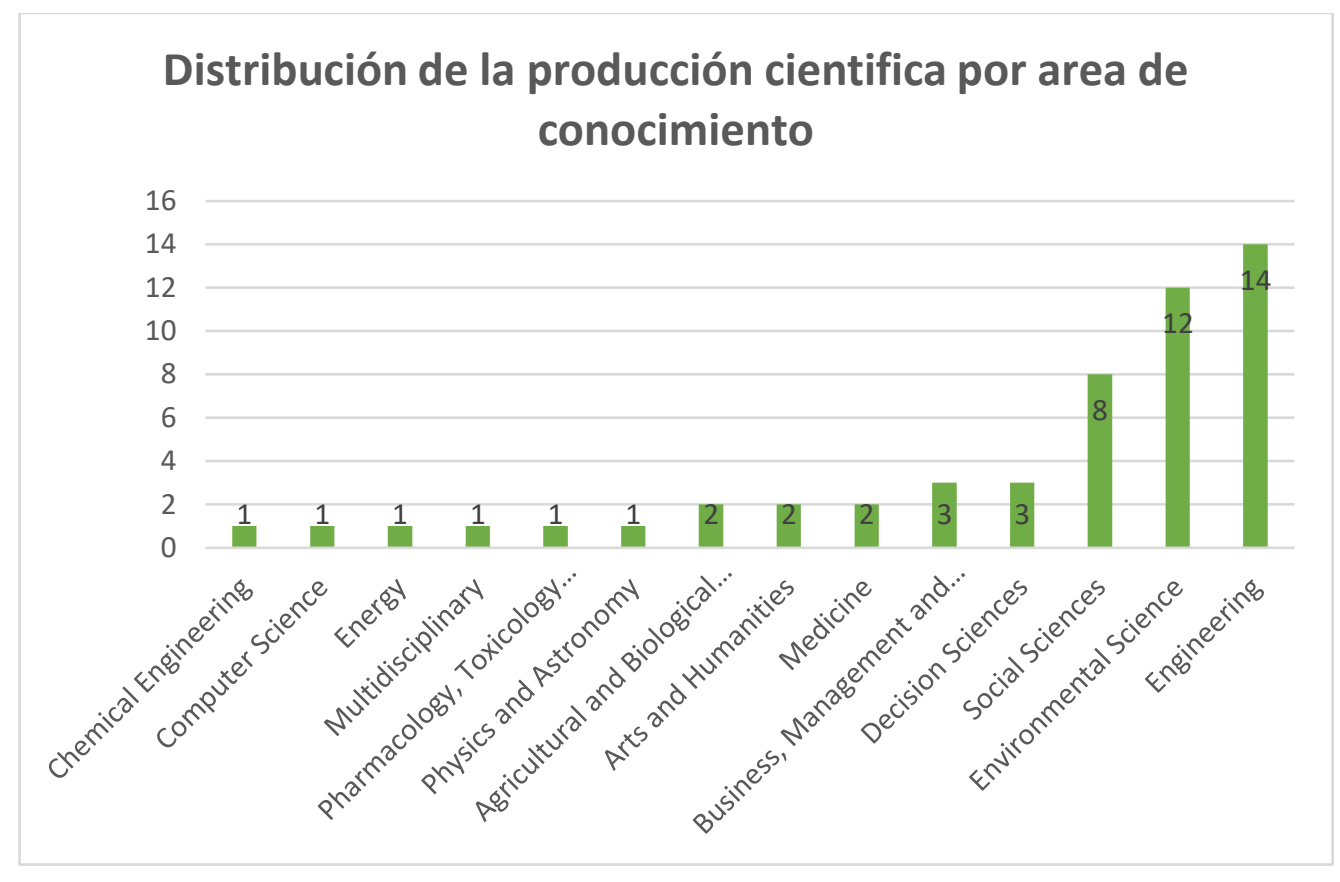

Fuente: Elaboración propia (2021); a partir de datos suministrados por Scopus. 
Ingeniería es el área de conocimiento con mayor número de aporte a través de las teorías que se enmarcan en ella, en la búsqueda del nuevo conocimiento sobre La Gestión de procesos internos en las empresas prestadoras de servicio de agua y alcantarillado con un total de 14 publicaciones, dentro de las cuales se encuentra "Propuesta de metamodelo para la regulación de la demanda urbana de agua potable" (Lizcano-Caro, Medina-Daza, \& González-Pérez, 2020) en este estudio consideran que los procedimientos ejecutan las empresas de acueducto y alcantarillado tienen igual importancia que la elaboración de presupuestos y la eficiencia económica, por lo que se propone un metamodelo que equilibre las inversiones eco ambientales con los criterios solidarios con el fin de determinar los procesos que se llevarán a cabo dentro de estas empresas para poder satisfacer la demanda urbana de agua potable. Por lo que, aunque no encontraron suficiente información sobre metamodelo al ser una práctica reciente llegaron a la conclusión que en estas herramientas también se deben tomar en cuenta las condiciones socioeconómicas de la comunidad donde se empleará el metamodelo para la optimización del servicio de acueducto.

Ciencias ambientales, es el segundo área de conocimiento con mayor número de publicaciones registrada con un total de 12 publicaciones, dentro de las cuales identificamos "Evaluación de la eficiencia de las empresas de agua y alcantarillado: un enfoque desagregado que contabiliza la calidad del servicio" (Molinos-Senante, MocholiArce, \& Sala-Garrido, Efficiency Assessment of Water and Sewerage Companies: a Disaggregated Approach Accounting for Service Quality, 2020) este estudio determina la calidad de las empresas de acueducto y alcantarillado evaluando el servicio que prestan y los procesos que se ejecutan a nivel interno en estas organizaciones. Este estudio dio como resultado que más de la mitad de las empresas tomadas para esta investigación cuentan con una eficiente prestación de servicio y la adecuación de sus procesos encontrándose diferencias entre las empresas privadas y las concesionarias que al final no fueron determinantes que influyeran en las variables que determinaban la calidad del servicio prestado y la gestión dentro de la empresa.

\subsection{Tipo de publicación}

La Figura 6 muestra como está distribuida la producción bibliografía según el tipo de publicación escogida por los autores. 
Figura 6. Tipo de publicación

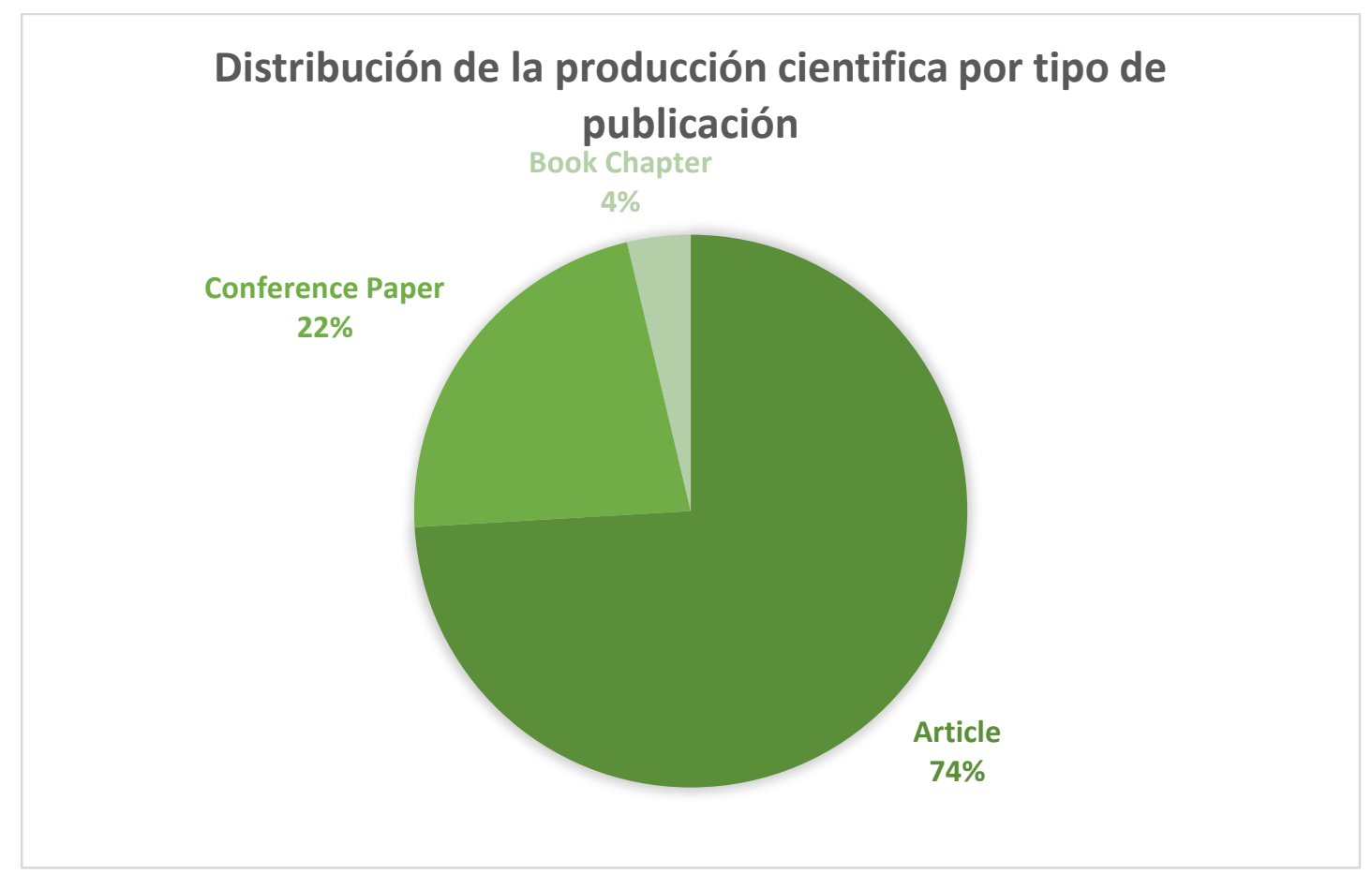

Fuente: Elaboración propia (2021); a partir de datos suministrados por Scopus.

Como lo muestra la Figura 6, dentro de los diferentes tipos de publicaciones, el 74\% del total de documentos identificados mediante la Fase 1 del Diseño Metodológico, corresponden a Artículos de Revista, el $22 \%$ pertenecen a las actas de conferencia dentro de las cuales se encuentra "“"Alcantarillados": Programa para el manejo integrado de sistemas de alcantarillado" (Castiblanco, Cortés, \& Valderrama, 2020) en esta investigación se propone "alcantarillados" un sistema operativo usado en los sistemas de alcantarillado y acueducto para simular el comportamiento hidráulico de una red de alcantarillado en condición de caudal no permanente, incluyendo diferentes condiciones de contorno, materiales, secciones transversales de conductos, pérdidas menores como manera de evaluar los procesos que se deben llevar a cabo para hacer posible la prestación de estos servicios.

Por último, los capítulos de libro representan el $4 \%$ del total de las publicaciones registradas que tiene relación con La Gestión de procesos internos en las empresas prestadoras de servicio de agua y alcantarillado, dentro de estos se encuentra "Una metodología para el mantenimiento de la red de alcantarillado hacia el cumplimiento de los Objetivos de Desarrollo Sostenible” (Penido, y otros, 2020) en este estudio se estudia 
la operación de las empresas de acueducto, Dicha operatividad está asegurada por rutinas de mantenimiento, tanto preventivas como correctivas, que son capaces de detectar puntos débiles donde pueden ocurrir pérdidas de efluentes y afectar cuerpos de agua, por lo que se estudian las cargas contaminantes que llegan al rio de las redes de alcantarillado y como la implementación de nuevos procesos puede ayudar a una operatividad encaminada a 3 de los 17 objetivos del desarrollo sostenible. Por lo que se concluye que el fortalecimiento de estos puntos débiles en las empresas de alcantarillado ayuda a una mejor apariencia y olor, así como niveles más altos de oxígeno disuelto lo que se traduce en la optimización de procesos y la calidad en el servicio prestado.

\section{CONCLUSIONES O CONSIDERACIONES FINALES}

Gracias al análisis bibliométrico propuesto en la presente investigación, se puede determinar que Colombia es el país de Latinoamérica con mayor número de registros bibliográficos en base de datos Scopus durante el periodo comprendido entre los años 2015 y 2020 con un total de 16 documentos. La producción científica relacionada al estudio de La Gestión de procesos internos en las empresas prestadoras de servicio de agua y alcantarillado, ha presentado un crecimiento importante durante el periodo anteriormente señalado, pasando de 2 publicaciones en 2015 a 9 unidades en 2020, es decir se logró un aumento en la creación de registros bibliográficos en un periodo de 5 años, lo que indica la importancia que los procesos internos de las empresas de acueducto y alcantarillado representan en la calidad del servicio prestado y en la eficiencia técnica de las plantas utilizadas para logar que el agua sea potable.

Uno de los aspectos importantes en la optimización de los procesos internos de las empresas de alcantarillado y de acueducto es el manejo de herramientas innovadoras y software que permitan relacionar mejor los datos recolectados tanto en el procedimiento técnico como el de atención al cliente, todo esto con el fin de garantizar la calidad en la prestación del servicio de agua potable y en el tratamiento de las aguas residuales en los servicios de alcantarillado en las zonas urbanas. La infraestructura que se utiliza en el tratamiento de estas aguas también está determinada por los procedimientos administrativos y las políticas empresariales implementadas para la innovación de las gestiones organizacionales determinando así las plantas utilizadas en estos procedimientos. Por lo anterior, se evidencia la importancia de los procesos internos que se desarrollan en las empresas de acueducto y alcantarillado en la calidad del servicio de 
acueducto en zonas urbanas, la maquinaria utilizada y la optimización de procesos técnicos, ya que gracias a estos procesos internos se determinan las políticas que se acogen en la gestión administrativa de estas compañías. Es por eso que se resalta la necesidad de estudios como el presentado en el presente documento, que realicen un recorrido por aquellos textos que aborden el mencionado tema, con el fin de dar al lector una visión amplia de la situación actual de la bibliografía sobre la Gestión de procesos internos en las empresas prestadoras de servicio de agua y alcantarillado.

\section{LISTA DE REFERENCIAS}

Birrichaga Gardida, D. (2006). Las empresa de agua dulce. Proyectos privados para sistemas de abastecimiento de agua potable en el Perú y México. Allpanchis, 3757.

Castiblanco, M., Cortés, G., \& Valderrama, J. (2020). “Alcantarillados": Programa para el manejo integrado de sistemas de alcantarillado. International Symposium on Hydraulic Structures - XXII Congreso Latinoamericano de Hidraulica. Guayana

Fontecha, J., Guaje, O., Duque, D., Akhavan-Tabatabaei, R., Rodríguez, J., \& Medaglia, A. (2019). Combined maintenance and routing optimization for large-scale sewage cleaning. Annals of Operations Research , 441 - 474.

García Rivera, C. Y. (2020). Problemática en la composición del Directorio en una Empresa Prestadora de. UNIVERSIDAD PERUANA DE CIENCIAS APLICADA.

Lizcano-Caro, J., Medina-Daza, R., \& González-Pérez, M. (2020). Proposed meta-model for the regulation of the urban demand for drinking water. Studia Romanica Posnaniensia, 35 - 54.

Lizcano-Caro, J., Medina-Daza, R., \& González-Pérez, M. (2020). Proposed meta-model for the regulation of the urban demand for drinking water. Tecnologia y Ciencias del Agua, 244 - 286.

Molinos-Senante, M., \& Maziotis, A. (2020). Technological and operational characteristics of the Chilean water and sewerage industry: A comparison of public, concessionary and private companies. Journal of Cleaner Production.

Molinos-Senante, M., \& Sala-Garrido, R. (2017). Decomposition of Productivity Growth of Water and Sewerage Companies: An Empirical Approach for Chile. Water Resources Managementv , 4309 - 4321. 
Molinos-Senante, M., M.-A. M., \& Sala-Garrido, R. (2020). Efficiency Assessment of Water and Sewerage Companies: a Disaggregated Approach Accounting for Service Quality. Water Resources Management , 4311 - 4328.

Penido, L., Amaral, K., Jorge, R., Metzger, J., Skroch, J., \& Gonçalves, R. (2020). A Methodology for Sewage Network Maintenance Toward the Fulfillment of Sustainable Development Goals. En World Sustainability Series (págs. 271 - 288).

Aldana, M. J. (2017). Integral network management: A case study of bogotá and the empresa de acueducto, alcantarillado y aseo de bogotá, EAB ESP. Paper presented at the Procedia Engineering, , 186 654-665. doi:10.1016/j.proeng.2017.03.282 Retrieved from www.scopus.com

Aldana, M. J., \& López, F. S. (2017). Water distribution system of bogotá city and its surrounding area, empresa de acueducto y alcantarillado de bogotá - EAB E.S.P. Paper presented at the Procedia Engineering, , 186 643-653. doi:10.1016/j.proeng.2017.03.281 Retrieved from www.scopus.com

Angarita, H., Niño, P., Vargas, D., Hernández, N., \& Torres, A. (2017). Identifying explanatory variables of structural state for optimum asset management of urban drainage networks: A pilot study for the city of bogota. [Identificación de factores de riesgo para la gestión patrimonial óptima de sistemas de drenaje urbano: Estudio piloto en la ciudad de Bogotá] Ingenieria e Investigacion, 37(2), 6-16. doi:10.15446/ing.investig.v37n2.57752

Arboleda, C. A. Á., \& Chicangana-Bayona, Y. A. (2015). Beginnig of the sewer in medellín (colombia), 1920-1955. [Inicios del alcantarillado en Medellín (Colombia), 1920-1955] HiSTOReLo, 7(14), 251-284. doi:10.15446/historelo.v7n14.47022

Buitrago-Guacaneme, A., Sotelo-Londoño, A., Pinilla-Agudelo, G. A., García-García, A., Moncada, L. I., \& Adler, P. H. (2018). Abundance and diversity of black flies (diptera: Simuliidae) in rivers of the andean eastern hills of bogotá (colombia), and its relationship with water stream physicochemical variables. [Abundancia e diversidade de moscas negras (Diptera: Simuliidae) em rios dos Cerros Orientais Andinos de Bogotá (Colômbia), e sua relação com variáveis físico-químicas das correntes de água] Universitas Scientiarum, 23(2), 291-317. doi:10.11144/Javeriana.SC23-2.aado 
Caro, J. A. L., Bolaños, S. J., \& Daza, R. J. M. (2019). Drinking water demand regulation system metamodel in long term horizons. [Metamodelo del sistema de regulación de la demanda de agua potable en horizontes de largo plazo] Ingeniare, 27(3), 361374. doi:10.4067/S0718-33052019000300361

Castiblanco, M. E. M., Cortés, G. A. H., \& Valderrama, J. G. S. (2020). “Alcantarillados”: Programa para el manejo integrado de sistemas de alcantarillado. Paper presented at the International Symposium on Hydraulic Structures - XXII Congreso Latinoamericano De Hidraulica, Retrieved from www.scopus.com

Castiblanco, P., Candil, D. C., Otalora, F., Alfonso, O. L. V., \& Diaz-Piraquive, F. N. (2018). Design of a prefeasibility model of costs for the construction of a veredal sewer in cundinamarca, for a medium company. Paper presented at the 2017 Congreso Internacional De Innovacion y Tendencias En Ingenieria, CONIITI 2017 - Conference Proceedings, , 2018-January 1-6. doi:10.1109/CONIITI.2017.8273357 Retrieved from www.scopus.com

Contreras, O. E., \& Rodríguez, L. T. (2015). A case on a case: Embedding sustainable entrepreneurship into a managerial-skills course. Paper presented at the Proceedings of the European Conference on Innovation and Entrepreneurship, ECIE, , 2015-January 139-146. Retrieved from www.scopus.com

Fontecha, J. E., Guaje, O. O., Duque, D., Akhavan-Tabatabaei, R., Rodríguez, J. P., \& Medaglia, A. L. (2020). Combined maintenance and routing optimization for large-scale sewage cleaning. Annals of Operations Research, 286(1-2), 441-474. doi:10.1007/s10479-019-03342-8

García-Ubaque, C. A., Ladino-Moreno, E. O., \& Zamudio-Huertas, E. (2020). Exploratory study on wetlands area decrease in bogota due to construction activity: 1950-2016. Revista Facultad De Ingenieria, 29(54) doi:10.19053/01211129.v29.n54.2020.10891

Garzón Duque, M., Ortiz Acosta, J., Tamayo Gaviria, N., \& Mesa Navas, V. (2018). Musculoskeletal disorders in sewer maintenance workers in an utility of colombia and its relationship to sociodemographic characteristics, employment and general medical conditions, medellin 2016. [Desordenes musculoesqueléticos en trabajadores de mantenimiento de alcantarillado en una empresa de servicios 
públicos de Colombia y su relación con características sociodemográficas, laborales y condiciones médicas generales, Medellín 2016] Revista De La Asociacion Espanola De Especialistas En Medicina Del Trabajo, 27(1), 17-28. Retrieved from www.scopus.com

Higuerey, A., Trujillo, L., \& González, M. M. (2017). Has efficiency improved after the decentralization in the water industry in venezuela? Utilities Policy, 49, 127-136. doi:10.1016/j.jup.2017.05.003

Lizcano-Caro, J. A., Medina-Daza, R., \& González-Pérez, M. G. (2020). Proposed metamodel for the regulation of the urban demand for drinking water. [Propuesta de metamodelo para la regulación de la demanda urbana de agua potable] Studia Romanica Posnaniensia, 47(3), 35-54. doi:10.14746/STROP.2020.473.003 